\title{
Total resection of an infected aortic arch aneurysm caused by Mycobacterium avium: a case report
}

\author{
Kei Yagami ${ }^{1 *}$ (D), Takashi Fujita', Shinichi Ishida ${ }^{1}$ and Masato Mutsuga ${ }^{2}$
}

\begin{abstract}
Background: Infected aortic arch aneurysms caused by Mycobacterium avium are rare in immunocompetent individuals. Promptly recognizing these aneurysms is important because delays in treatment result in aneurysm rupture and a high fatality rate. Although Salmonella species, Streptococcus species, Staphylococcus aureus, and S. epidermis are commonly found in immunocompetent individuals, to our knowledge, infected aortic arch aneurysms caused by M. avium have not yet been reported.

Case presentation: We report the case of a 63-year old immunocompetent man who underwent total arch replacement following infection by the nontuberculous mycobacteria M. avium. The procedure involved total aneurysmal resection and arch replacement with a rifampicin-bonded gelatin-sealed woven Dacron graft. He was discharged without complications and remained asymptomatic after 30 months.

Conclusion: In this brief report, we outline and discuss the rare successful case of total arch replacement using total aneurysmal resection and rifampicin-bonded gelatin-sealed woven Dacron graft for an infected aortic arch aneurysm resulting from $M$. avium in an immunocompetent patient.
\end{abstract}

Keywords: Nontuberculous mycobacteria, Mycobacterium avium, Infected aneurysm, Aortic arch aneurysm, Total arch replacement, Rifampicin-bonded gelatin-sealed woven Dacron graft

\section{Background}

Infected aortic arch aneurysms caused by Mycobacterium avium are very rare in immunocompetent individuals $[1,2]$. Promptly recognizing these aneurysms is important because delays in treatment results in aneurysm rupture and a high fatality rate. Although Salmonella species, Streptococcus species, Staphylococcus aureus, and S. epidermis are commonly found in immunocompetent individuals, to our knowledge, infected aortic arch aneurysms caused by $M$. avium and their successful repair have not yet been reported. Here, we report a rare case of total arch replacement using a rifampicin-bonded gelatin-sealed woven Dacron graft for an infected aortic arch aneurysm resulting from M. avium.

\footnotetext{
*Correspondence: yagamikei2002@yahoo.co.jp

${ }^{1}$ Gifu Prefectural Tajimi Hospital Cardiac Surgery, 161-5, Maebata-cho,

Tajimi-City, Gifu 507-8532, Japan

Full list of author information is available at the end of the article
}

\section{Case presentation}

The patient was a 63-year-old man who was diagnosed with malignant lymphoma in 2013 and underwent 2 years of radiotherapy and chemotherapy, after which he was deemed to be in complete remission. During the course of chemotherapy, the patient's white blood cell count reduced to $200 / \mu l$, and he was in an immunocompromised state, but at the end of the chemotherapy, the count recovered to normal, and the patient lived a normal life without any medication. In 2016, mediastinal lymphadenopathy was noted on a follow-up chest computed tomography $(\mathrm{CT})$, and his history revealed a dry cough for several months. Therefore, bronchoscopic lymph node biopsy, sputum culture, and blood culture were performed. Although the blood culture was negative, sputum culture grew $M$. avium, and he was diagnosed with nontuberculous mycobacterial infection and started on a course of rifampicin $570 \mathrm{mg} /$ day, ethambutol $750 \mathrm{mg} /$ day, and clarithromycin $800 \mathrm{mg} /$ day.

(C) The Author(s). 2019 Open Access This article is distributed under the terms of the Creative Commons Attribution 4.0 International License (http://creativecommons.org/licenses/by/4.0/), which permits unrestricted use, distribution, and 
After 1 month, he developed low-grade fever and chest pain, and a chest CT revealed a new saccular aneurysm of the aortic arch measuring $41 \times 49 \mathrm{~mm}$ (Fig. 1a). He was continued on antibiotic treatment and closely followed; however, after 1 month a follow-up chest CT revealed that the saccular aneurysm had expanded to $43 \times 62 \mathrm{~mm}$ (Fig. 1b), suggesting false aneurysm formation. 18F-fluorodeoxyglucose (FDG) positron emission tomography with CT (PET/CT) was also performed for suspected infective aneurysm, and this revealed FDG uptake in the aneurysm (Fig. 1c). Therefore, the patient was diagnosed with an infected aortic arch aneurysm due to M. avium.

Early surgical repair was deemed necessary because of the rapid expansion of the aneurysm. The options available were total arch replacement or thoracic endovascular aortic repair (TEVAR) with one or two debranching arch vessels as options. However, we opted to proceed with total arch replacement using a rifampicin-bonded gelatinsealed woven Dacron graft because we strongly suspected an infective etiology. At surgery, median sternotomy was performed, and cardiopulmonary bypass was established via the femoral artery and the superior and inferior vena cava cannulas. Systemic anticoagulation was initiated with 300 units $/ \mathrm{kg}$ heparin. Under hypothermic circulatory arrest at a pharyngeal temperature of $23^{\circ} \mathrm{C}$ and a rectal temperature of $25^{\circ} \mathrm{C}$, the aortic arch was opened, and balloon-tipped selective cerebral perfusion cannulas were inserted. Balloon-occluded distal anastomosis was then performed under femoral perfusion of the lower body. The aneurysm was extensively debrided, and the resected section was sent for culture. Finally, the aortic arch was replaced with a rifampicin-bonded gelatin-shield 30-mm 4 branch woven Dacron graft (J Graft Shield Neo; Japan Lifeline Co., Ltd., Japan). Because of the presence of infected aneurysms near the origin of left subclavian artery, we could not perform in situ reconstruction of the artery and performed only extraanatomical reconstruction. The origin of left subclavian artery was closed, and the reconstruction was peripherally performed.

Postoperatively, no major complications were observed, and CT at the 14th postoperative day confirmed complete resection of the aneurysm (Fig. 2). M. avium was isolated from the resected tissue. The patient was discharged on the 15th postoperative day without incident and continued on the course of rifampicin $570 \mathrm{mg} /$ day, ethambutol $750 \mathrm{mg} /$ day, and clarithromycin $800 \mathrm{mg} /$ day. He has received oral antibiotic therapy as an outpatient for 30 months with no symptoms, and we plan to continue them for several more months.

\section{Discussion and conclusions}

Nontuberculous mycobacteria are rarely pathogenic in immunocompetent individuals, but they are important pathogens in immunocompromised individuals [1, 2]. In immunocompetent individuals, aneurysm infections are typically with Salmonella species, Staphylococcus aureus, S. epidermis, or Streptococcus species. In our case, the patient had previously undergone radiotherapy and chemotherapy for malignant lymphoma, so will have developed a
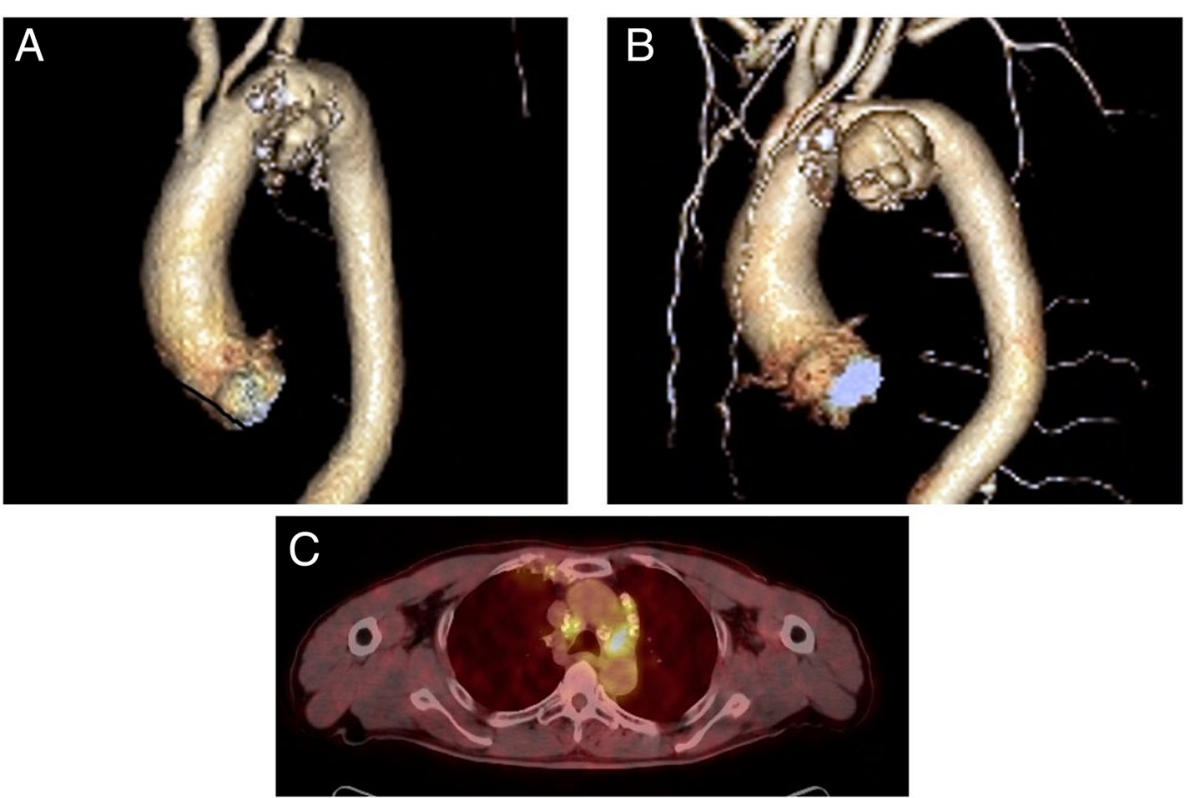

Fig. 1 a Computed tomography (CT) of the chest revealed a new saccular aneurysm of the aortic arch. b After 1 month, follow-up CT showed a rapidly expanding saccular aneurysm of the aortic arch. c F-18 fluorodeoxyglucose positron emission tomography with computed tomography showing intense fluorodeoxyglucose avidity in soft tissue involving the aneurismal segment of the aortic arch 


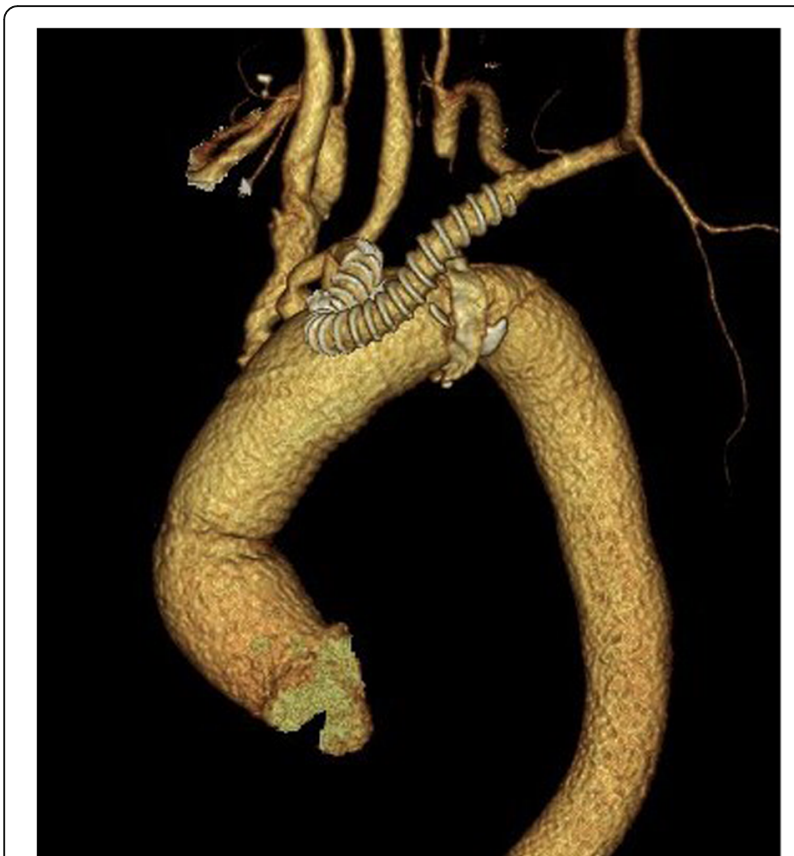

Fig. 2 Postoperative computed tomography (CT) showed no aneurysm of the aortic arch and complete resection of the lesion

temporary immunocompromised status that could have accounted for his rare presentation.

CT is the standard tool for imaging suspected infected aneurysms. It provides a detailed assessment of the aneurysm and can offer morphological and anatomical images in high resolution. Periaortic fat, contrast enhancement, or fluid collections are clues to infection, but such features are not always prominent and may be missed in lowgrade infection. In suspected cases that cannot be diagnosed by CT, diagnosis can be confirmed by 18F-FDG PET/CT [3], as in the current report.

TEVAR has been reported to have good results in the treatment of infected aneurysms. However, the risks of repeat infection are high after repair, not least because it requires that the stent graft be implanted in an infected aorta, and the procedure can be used for bridging to surgical repair [4]. Consequently, several permanent approaches have been reported to treat infected aortic aneurysms that use rifampicin-bonded grafts, homografts, or omental wrapping. Raman et al. reported an excellent outcome when using a rifampicin-bonded in situ graft for an infected abdominal aortic aneurysm due to M. avium in an immunocompromised patient [1]. Their patient was discharged on the seventh postoperative day and remained asymptomatic after 2 years of follow-up. In contrast, Tsilimparis et al. reported the use of a homograft for an infected abdominal aortic aneurysm caused by $M$. avium in an immunocompromised patient [2]. However, their patient developed multiorgan failure and had care withdrawn on the second postoperative day. Uchida et al. reported excellent results with the use of rifampicin-bonded and omental pedicle grafts in 23 cases of infected thoracic, thoracoabdominal, or abdominal aortic aneurysms [5], but their procedure was for mycotic rather than nontuberculous mycobacterial aneurysms. Some reports have reported good results with prosthesis made from bovine pericardium in the setting of infection [6].

It is difficult to prepare homografts at the time of surgery in Japan. It was also considered difficult to use the omentum in the present case because the patient had previously undergone abdominal surgery, and we considered that the aortic arch replacement using the bovine pericardium was morphologically difficult. Therefore, we considered open surgical repair with aneurysmal resection and rifampicin-bonded grafting as better realistic therapy. At present, total resection of the infected aneurysmal wall and replacement with a rifampicin-bonded graft, with or without an omental pedicle, may be the optimal therapy. $M$. avium infection of an aneurysm in the thoracic aortic is rare in immunocompetent patients. We report the diagnosis in a patient who was successfully treated with a rifampicin-bonded gelatin-shield woven Dacron graft. Meticulous follow-up is mandatory for these cases, and therapeutic experience needs to be accumulated.

\section{Abbreviations}

CT: Computed tomography; FDG: Fluorodeoxyglucose; PET/CT: Positron emission tomography with computed tomography; TEVAR: Thoracic endovascular aortic repair

\section{Acknowledgments}

Not applicable.

\section{Authors' contributions}

All authors participated in the design of the case report and coordination and helped to the draft the manuscript. All authors have read and approved the final manuscript.

\section{Funding}

The authors have no funding, financial relationships, or interest to disclose.

Availability of data and materials

Not applicable.

Ethics approval and consent to participate

Hospital Ethics committee approved the study.

\section{Consent for publication}

Not applicable.

\section{Competing interests}

The authors declare that they have no competing interests.

\section{Author details}

${ }^{1}$ Gifu Prefectural Tajimi Hospital Cardiac Surgery, 161-5, Maebata-cho, Tajimi-City, Gifu 507-8532, Japan. ${ }^{2}$ Department of Cardiac Surgery, Nagoya University Graduate School of Medicine, Nagoya, Japan. 
Received: 21 June 2019 Accepted: 13 August 2019

Published online: 19 August 2019

\section{References}

1. Raman SR, Pokala L, Jamil Z. Nontuberculous mycobacterial abdominal aortic aneurysm in a patient with AIDS. Am Surg. 2010;76:1031-3.

2. Tsilimparis N, Defreitas D, Debus ES, Reeves JG. Latent Mycobacterium avium infection causing a mycotic suprarenal aortic aneurysm in a human immunodeficiency virus-positive patient. Ann Vasc Surg. 2014;28:1035.e1-4.

3. Murakami M, Morikage N, Samura M, Yamashita O, Suehiro K, Hamano K. Fluorine-18-fluorodeoxyglucose positron emission tomography-computed tomography for diagnosis of infected aortic aneurysms. Ann Vasc Surg. 2014;28:575-8.

4. Kritpracha B, Premprabha D, Sungsiri J, Tantarattanapong W, Rookkapan S, Juntarapatin P. Endovascular therapy for infected aortic aneurysms. J Vasc Surg. 2011;54:1259-65.

5. Uchida N, Katayama A, Tamura K, Miwa S, Masatsugu K, Sueda T. In situ replacement for mycotic aneurysms on the thoracic and abdominal aorta using rifampicin-bonded grafting and omental pedicle grafting. Ann Thorac Surg. 2012;93:438-42.

6. Chida Y, Shiroto K, Kadohama T, Yamaura G, Takagi D, Aida H, Kagaya S, Yamamoto H. In situ replacement with bovine pericardial roll graft for infected aneurythm of the toracoabdominal aorta. Kyobu Geka. 2015;68: 976-9.

\section{Publisher's Note}

Springer Nature remains neutral with regard to jurisdictional claims in published maps and institutional affiliations.

Ready to submit your research? Choose BMC and benefit from:

- fast, convenient online submission

- thorough peer review by experienced researchers in your field

- rapid publication on acceptance

- support for research data, including large and complex data types

- gold Open Access which fosters wider collaboration and increased citations

- maximum visibility for your research: over $100 \mathrm{M}$ website views per year

At BMC, research is always in progress.

Learn more biomedcentral.com/submissions 